\title{
Magnetically Induced Ring-Current Strengths in Möbius Twisted Annulenes
}

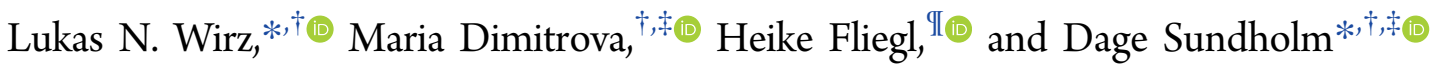 \\ ${ }^{\dagger}$ Department of Chemistry, University of Helsinki, P.O. Box 55, A. I. Virtasen aukio 1, FIN-00014 Helsinki, Finland \\ ${ }^{\ddagger}$ Hylleraas Centre for Quantum Molecular Sciences, Department of Chemistry, University of Oslo, P.O. Box 1033 Blindern, N-0315 \\ Oslo, Norway \\ ${ }^{\mathrm{I}}$ Centre for Advanced Study at the Norwegian Academy of Science and Letters, Drammensveien 78, N-0271 Oslo, Norway
}

\section{Supporting Information}

ABSTRACT: The topology of twisted molecular rings is characterized by the linking number, which is equal to the sum of the twist-a local property of the molecular frame-and the writhe- a global parameter, which represents the bending of the molecular ring. In this work, we investigate a number of cyclic all-trans $\mathrm{C}_{40} \mathrm{H}_{40}$ annulenes with varying twisting numbers for a given linking number and their dications. The aromatic character is assessed by calculating ring-current strength susceptibilities using the gauge-including magnetically induced currents (GIMIC) method, which makes it possible to conduct a systematic study of the relation between the topology and aromaticity of twisted molecules. We found that the aromatic properties of the investigated Möbius twisted molecules are not only dependent on the linking number as previously suggested but also depend strongly on the partitioning of the linking number into the twist and writhe contributions.

$\mathrm{I}_{\mathrm{d}}^{\mathrm{n}}$ $\mathrm{n}$ topology, a closed ribbon that is not a knot is fully characterized by its integer linking number $L_{k}$, which can intuitively be understood as the number of half twists that one end of an open ribbon has to undergo before it meets the other end to form a closed ribbon loop. Closed ribbons with an even $L_{k}$ have two sides and two edges, whereas those with odd $L_{k}$, such as the well-known singly twisted Möbius strip with $L_{k}=$ \pm 1 , have only one side and one edge.

A given closed ribbon can be embedded into the threedimensional (3D) Cartesian space in an infinite number of ways. Two properties that characterize, but not uniquely define, such an embedding are the writhe, or writhing number $\left(W_{r}\right)$, and the twist $\left(T_{w}\right)^{1,2} W_{r}$ is a nonlocal property of a closed curve in $3 \mathrm{D}$, which by convention is the curve lying in the middle of the ribbon. ${ }^{3}$ In contrast, $T_{w}$ is the path integral of the local twist around the central curve. ${ }^{3}$ The linking number in eq 1 is obtained as the sum of the twist and writhe of the closed ribbon with a given embedding. ${ }^{4-6} T_{w}$ and $W_{r}$ can take any real value, whereas the linking number is an integer. The twist, writhing, and linking numbers multiplied by $\pi$ yield the corresponding angles in radians.

$$
L_{k}=T_{w}+W_{r}
$$

The 3D embedding of a closed ribbon is always chiral when either $L_{k}, T_{w}$, or $W_{r}$ is nonzero because these parameters can take the same positive or negative values. Equation 1 shows that closed ribbons with nonzero $L_{k}$ are always chiral, while ribbons with $L_{k}=0$ may be achiral. Ribbons with $L_{k}=0$ are achiral only when $T_{w}=W_{r}=0$, which holds for planar rings without any local twists, such as benzene.
The energy levels of Möbius twisted annulenes and aromaticity rules can be qualitatively obtained by diagonalizing the Hückel molecular orbital (HMO) Hamiltonian for the molecular ring. ${ }^{1,7-9}$ For rings with even $L_{k}$ numbers, the wellknown $(4 n+2)$ Hückel rule for aromaticity holds at the HMO level, ${ }^{10,11}$ whereas when $L_{k}$ is an odd integer, molecules with $4 n$ $\pi$ electrons are aromatic, ${ }^{7,12}$ which is valid only for monocyclic systems. The opposite rules hold for antiaromatic molecules, sustaining paratropic ring currents. ${ }^{13-17}$ It has been assumed that aromatic properties are mostly independent of the partitioning, that is, the $L_{k}$ value determines the aromaticity of Möbius twisted molecular rings. ${ }^{1,2,7-9,18-25}$ However, in order to incorporate $T_{w}$ and $W_{r}$ effects, one has to go beyond the HMO approximation because the HMO Hamiltonian does not contain any information about the splitting of $L_{k}$ into $T_{w}$ and $W_{r}$.

We chose all-trans annulenes as model systems because they have roughly the correct bonding angles for large annulene rings. The initial molecular structures of the annulenes with varying twist and writhe were generated by first constructing a circular polygon line in 3D. Depending on the chosen writhe, it is twisted around the $y$ axis, compressed along the $x$ axis, and rescaled to keep the total length constant. This backbone approximately defines the writhe but not the twist or linking number of the final structure. The atoms are then incrementally placed such that they are uniformly twisted around the backbone. Finally, we employ a primitive force field that

Received: February 9, 2018

Accepted: March 13, 2018

Published: March 13, 2018 


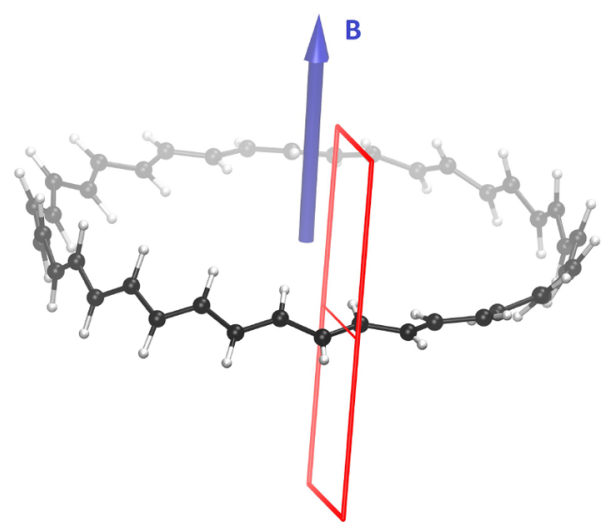

(a)

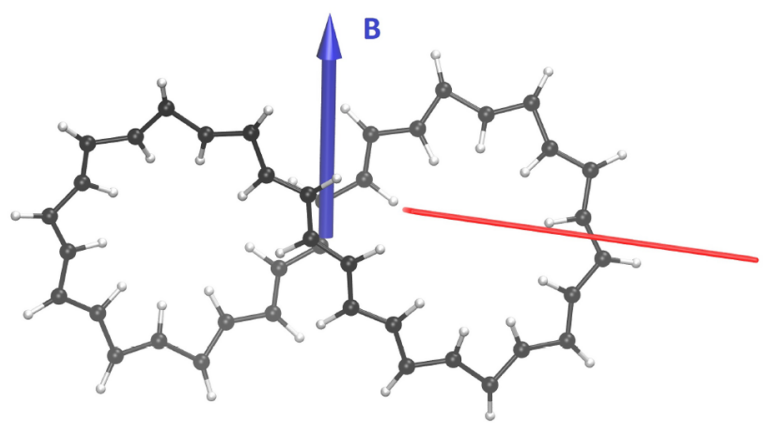

(b)

Figure 1. Molecular structure of the neutral molecule with $L_{k}=2$ having (a) the largest and (b) the smallest $T_{w}$ value. The integration plane for the calculation of the ring-current strength is indicated in red, and the blue arrow is the magnetic field vector $B$.

considers only bond lengths, bond angles, and dihedrals. The structural changes due to the force field optimization are very small.

The initial molecular structures of the neutral and doubly charged molecules were subsequently optimized at the density functional theory (DFT) level using the BP86 functional as implemented in Turbomole. ${ }^{26-30}$ The $\operatorname{def} 2-\mathrm{SV}(\mathrm{P})$ basis sets and the $\mathrm{m} 3$ grid were used in the structural optimization. ${ }^{31,32}$ Constraints were imposed in order to avoid relaxation to the same lowest-energy structure during the course of the optimization. The $\mathrm{C}-\mathrm{C}$ and $\mathrm{C}-\mathrm{H}$ distances of the initial structures were optimized, while all angular degrees of freedom were frozen. When the constrained optimizations converged, the Cartesian coordinates of the carbon atoms were fixed and the positions of the hydrogen atoms were fully optimized. The optimization procedure yielded molecular geometries with the same $L_{k}$ number and about the same $T_{w}$ and $W_{r}$ values as the initial structures. The exact $T_{w}$ and $W_{r}$ values were determined for the optimized molecular geometries. The final structures for the neutral molecule with $L_{k}=2$ and the largest and smallest $T_{w}$ values are shown in Figure 1. The other $L_{k}=2$ structures are intermediates between the two extremes. The $L_{k}=0$ and 1 structures are largely the nontwisted and singly twisted versions of the $L_{k}=2$ ones. The Cartesian coordinates of the studied molecules are given in the Supporting Information.

The nuclear magnetic shielding constants were calculated at the BHLYP level using the def2-SVP basis sets and the $\mathrm{m} 5$ integration grid. ${ }^{31-36}$ The magnetically induced current densities were calculated with the gauge-including magnetically induced current (GIMIC) method using basis set information as well as the one-electron atomic orbital (AO) density matrix and the one-electron magnetically perturbed $\mathrm{AO}$ density matrices obtained in calculations of nuclear magnetic shielding constants at the DFT level. ${ }^{37-41}$ Ring-current strength susceptibilities were obtained by integrating the current-density flow that passes a plane that intersects the molecular ring, as shown in Figure 1. In the current-density calculations, the molecular ring was oriented in order to maximize the projected area of the molecular ring perpendicularly to the external magnetic field because it is expected to yield the strongest ring current. ${ }^{42-44}$ Figure 1 shows that the area of the molecular loop projected in the direction of the magnetic field decreases with the absolute value of $W_{r}$, which might be a contributing reason for the decreasing current strengths for molecules with higher
$W_{r}$ values. The figures were prepared using Gnuplot, VMD, Paraview, and GIMP. ${ }^{45-48}$ The line integral convolution method for the visualization of vector fields from Paraview was employed. $^{49}$

The BHLYP functional has been employed in the currentdensity calculations because previous current-density calculations showed that DFT calculations at the B3LYP level have a tendency to overestimate the degree of antiaromaticity for strongly antiaromatic molecules, ${ }^{50}$ whereas B3LYP calculations yield an accurate degree of aromaticity for aromatic molecules. $^{51,52}$ Current-density calculations on antiaromatic porphyrinoids using the BHLYP functional yield ring-current strengths that are in good agreement with the values obtained at the second-order Møller-Plesset (MP2) level.

The generalized Hückel rules for Möbius molecules state that monocyclic annulenes with an even linking number are aromatic when they have $(4 n+2) \pi$ electrons, and annulenes with odd $L_{k}$ values are aromatic when they have $4 n \pi$ electrons. The opposite rules hold for Möbius twisted antiaromatic molecular monocycles. Thus, according to the generalized Hückel rules, the aromatic character of a twisted molecular ring should be independent of the partitioning of $L_{k}$ between the twist $T_{w}$ and writhe $W_{r}$. One has to go beyond Hückel theory in order to investigate how the aromatic character depends on $T_{w}$ and $W_{r}$ for a given linking number. Because there is a linear relation between hydrogenation enthalpies of aromatic molecules and the strengths of magnetically induced ring currents, ${ }^{53}$ we use the ring-current strengths for systematically assessing the aromatic character of a number of Möbius twisted all-trans $\mathrm{C}_{40} \mathrm{H}_{40}$ annulenes with $L_{k}$ values of 0,1 , and 2 and varying $T_{w}$.

The ring-current strength as a function of the $T_{w}$ value for the $L_{k}=0$ annulenes is shown in Figure 2. The aromatic doubly charged, and the antiaromatic neutral all-trans $\mathrm{C}_{40} \mathrm{H}_{40}$ annulenes exhibited lowest energy when $L_{k}=T_{w}=W_{r}=0$. For small $T_{w}$ values, the neutral all-trans $\mathrm{C}_{40} \mathrm{H}_{40}$ annulene is expectedly antiaromatic, sustaining a paratropic ring current of $-17 \mathrm{nA} / \mathrm{T}$, which is about $50 \%$ stronger than the diatropic ring-current strength of benzene. ${ }^{54}$ By twisting the molecular ring and compensating for the twist by introducing writhe, the $\mathrm{C}_{40} \mathrm{H}_{40}$ ring becomes less antiaromatic. There is a nearly linear relation between the ring-current strength and the $T_{w}$ value, as shown in Figure 2. For the molecular structure with $T_{w}=-1.68$ and $W_{r}=1.68$, the ring is nonaromatic, sustaining a ring current 


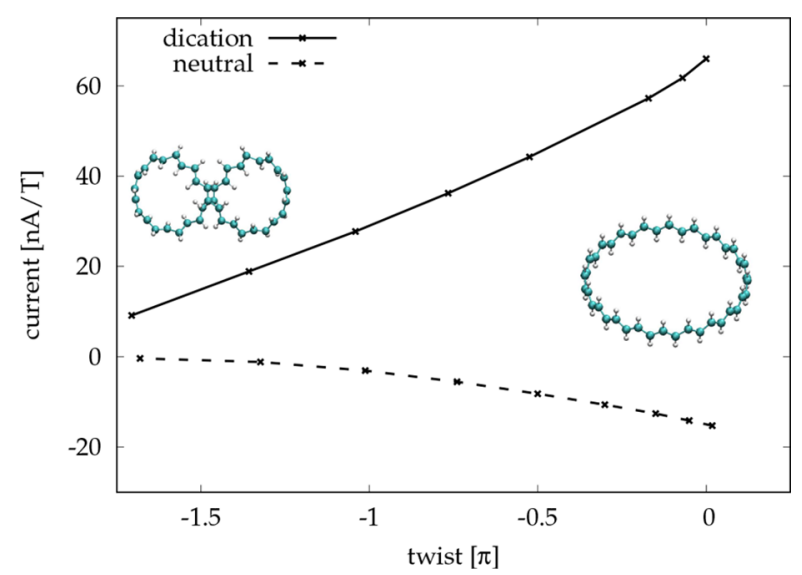

Figure 2. Ring-current strengths as a function of the twist number $T_{w}$ of the neutral, and the doubly charged dication of all-trans $\mathrm{C}_{40} \mathrm{H}_{40}$ annulene with $L_{k}=0$. The topology for the two extreme structures is also shown.

of only $-0.4 \mathrm{nA} / \mathrm{T}$. The optimized geometry of the doubly charged $\mathrm{C}_{40} \mathrm{H}_{40}$ dication is planar. It is strongly aromatic, sustaining a ring current of $66 \mathrm{nA} / \mathrm{T}$, as also predicted from the Hückel rule. Twisting the molecule leads to a weaker aromatic character. For the ring with $T_{w}=-1.7$, the ring-current strength is $9.4 \mathrm{nA} / \mathrm{T}$, illustrating how changes in the twist significantly affect the aromatic character.

The aromatic character of the singly twisted Möbius annulenes $\left(L_{k}=1\right)$ follows the predictions of the generalized Hückel rule. The neutral singly twisted $\mathrm{C}_{40} \mathrm{H}_{40}$ ring with $4 n \pi$ electrons is strongly aromatic, sustaining a net ring current of $38 \mathrm{nA} / \mathrm{T}$, whereas the doubly charged one is strongly antiaromatic with a magnetically induced ring-current strength of $-45 \mathrm{nA} / \mathrm{T}$. The lowest energy is obtained for the least deformed molecular structures with the largest $T_{w}$ value. The $T_{w}$ and $W_{r}$ values of the most twisted neutral and dicationic annulenes with $L_{k}=1$ are 1.13 and -0.13 , respectively. The ring-current strengths diminish systematically when the twist is transferred to writhe. The molecules with the largest inspected $W_{r}$ value of 1.42 sustain ring currents with strengths of 3.4 and $-13.6 \mathrm{nA} / \mathrm{T}$, for the neutral and dicationic ring, respectively. There is a nearly linear relation between the ring-current strength and the $T_{w}$ value, except in the vicinity of $T_{w}=1$, where the degree of aromaticity and antiaromaticity increases rapidly for larger twists. The ring-current strength as a function of the $T_{w}$ value is shown for the $L_{k}=1$ species in Figure 3. A 3D vector plot of the current densities is shown in Figure 4.

The doubly twisted annulenes also obey the generalized Hückel rules. The neutral doubly twisted $\mathrm{C}_{40} \mathrm{H}_{40}$ rings with $4 n$ $\pi$ electrons are antiaromatic, and the corresponding dications are aromatic. The most twisted and least deformed rings sustain the strongest ring currents. The ring-current strength for the most twisted neutral ring with a $T_{w}$ value of 2.31 sustains a paratropic ring current of $-8.4 \mathrm{nA} / \mathrm{T}$. The corresponding dication is strongly aromatic, sustaining a diatropic ring current of $57 \mathrm{nA} / \mathrm{T}$. The most deformed $L_{k}=2$ rings with a $T_{w}$ value of 0.66 are almost nonaromatic, sustaining ring currents of -1.4 and $2.4 \mathrm{nA} / \mathrm{T}$ for the neutral and doubly charged rings, respectively. The degree of antiaromaticity of the neutral ring decreases nearly linearly with decreasing $T_{w}$ value. For the dication, the degree of aromaticity increases rapidly with increasing $T_{w}$ value, especially when $T_{w}$ is larger than 2 . The ring-current strength as a function of the $T_{w}$ value is shown for

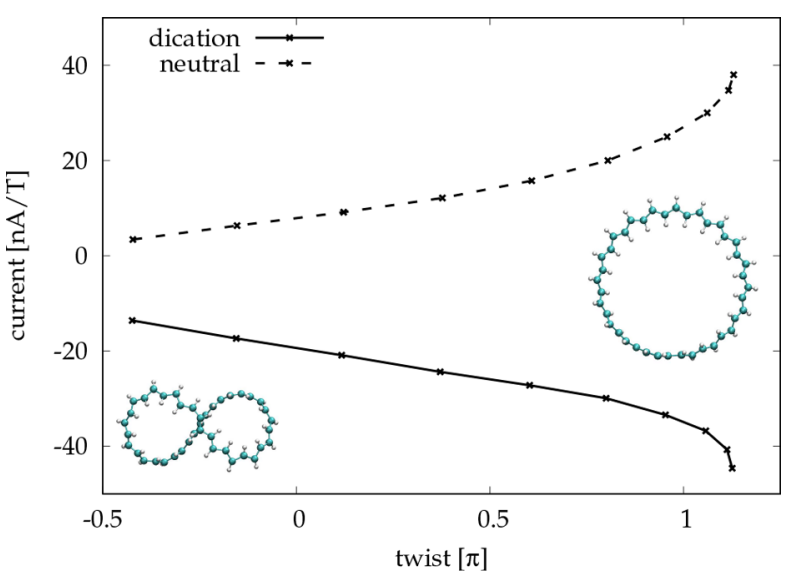

Figure 3. Ring-current strengths as a function of the twist number $T_{w}$ of the singly twisted neutral, and the doubly charged dication of alltrans $\mathrm{C}_{40} \mathrm{H}_{40}$ annulene with $L_{k}=1$. The topology for the two extreme structures is also shown.

the $L_{k}=2$ species in Figure 5. The lowest energy is obtained for the least deformed molecular structures with the largest $T_{w}$ value and the strongest ring current. An interaction between the two strands can be seen in Figure 6, where the current flow entwines in the central region of the molecule.

The molecules with the smallest writhe are energetically the most stable ones, which also holds for both the neutral and dicationic species. The total energies as a function of $T_{w}$ are given in the Supporting Information. The neutral molecules and the dications have almost constant gaps between the energies of the highest occupied molecular orbital (HOMO) and the lowest unoccupied molecular orbital (LUMO). The HOMO-LUMO gaps depend very little on the topology and the employed functional. They are somewhat larger for the aromatic rings sustaining strong ring currents than for the ones with weaker ring currents. For the antiaromatic rings, the HOMO-LUMO gap is slightly smaller for the molecules sustaining strong paratropic ring currents than that for the ones with weak paratropic ring currents. Even though the differences in the HOMO-LUMO gaps are small, the studied molecules follow the general relation between ring-current strengths and HOMO-LUMO gaps. ${ }^{55}$ Values for the HOMO-LUMO gaps calculated at the BHLYP and HF levels as well as the harmonic oscillator model of aromaticity (HOMA) index ${ }^{56}$ are reported in the Supporting Information.

In this work, we have determined relations between the degree of aromaticity and molecular topology by systematically investigating a set of neutral and doubly charged all-trans $\mathrm{C}_{40} \mathrm{H}_{40}$ annulenes using first-principle computational methods. In order to assess the degree of aromaticity, we calculated magnetically induced ring-current strength susceptibilities at the DFT level using the GIMIC method. The obtained ringcurrent strengths provide a reliable indicator for the aromatic character. Molecular rings with different topology were constructed with given $L_{k}$ numbers of 0,1 , and 2 by varying $T_{w}$. The aromatic character as judged from the direction and strength of the magnetically induced ring currents was determined as a function of $T_{w}$ for neutral and doubly charged molecular rings. The calculations show that the generalized Hückel rules for Möbius twisted molecules qualitatively hold. However, the degree of aromaticity depends strongly on the partitioning of the linking number $\left(L_{k}\right)$ between the twist $\left(T_{w}\right)$ and the writhe $\left(W_{r}\right)$. The strongest ring currents were obtained 


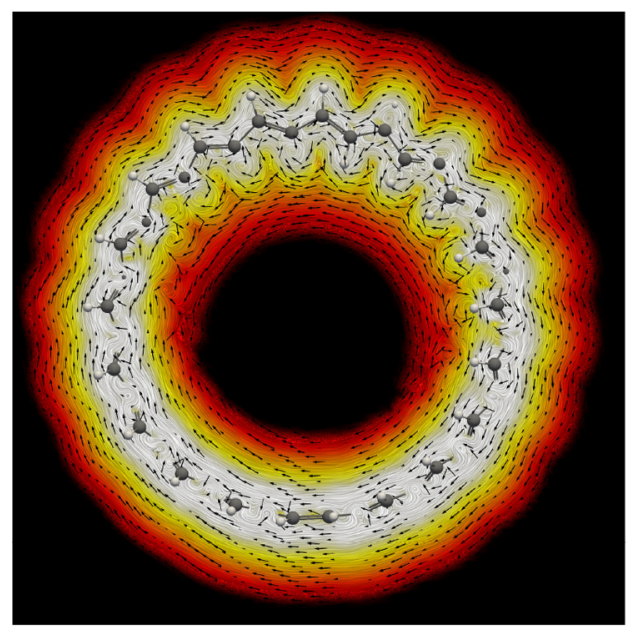

(a)

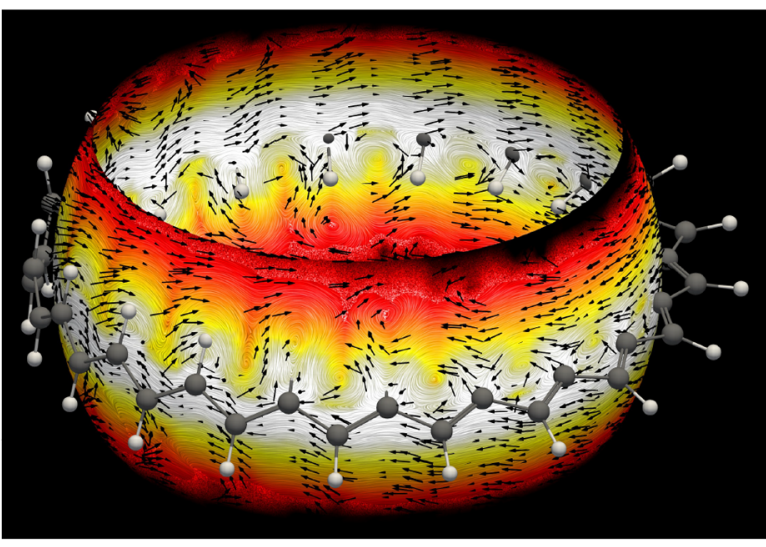

(b)

Figure 4. Current-density vector field illustrated using the line integral convolution (LIC) method for the neutral aromatic molecule with $L_{k}=1$ and twist number $T_{w}=1.13$. (a) Horizontal slice perpendicular to the magnetic field. (b) Slice on a spherical surface. More LIC pictures are given in the Supporting Information.

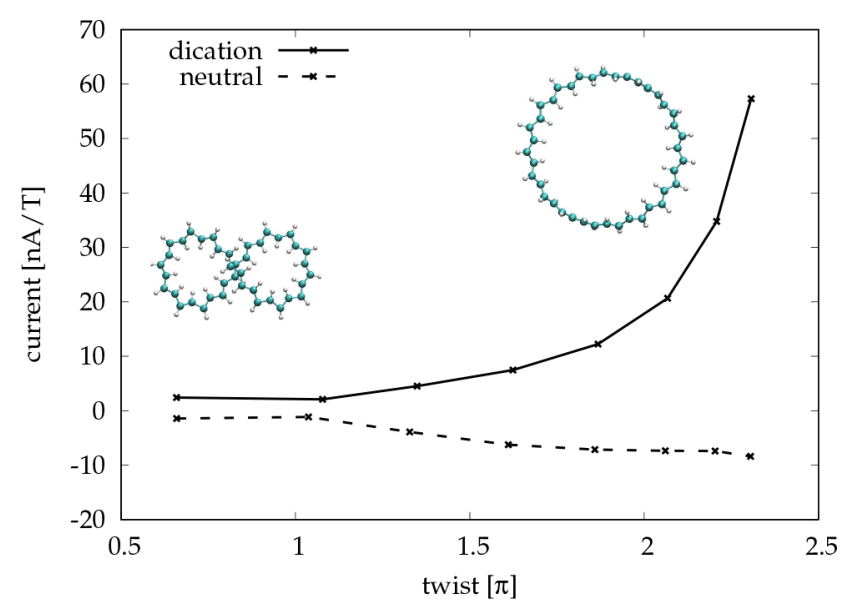

Figure 5. Ring-current strengths as a function of the twist number $T_{w}$ of the doubly twisted neutral and doubly charged dication of all-trans $\mathrm{C}_{40} \mathrm{H}_{40}$ annulene with $L_{k}=2$. The topology for the two extreme structures is also shown. for the most twisted molecules with the smallest $W_{r}$. The ringcurrent strength declines rapidly with increasing $W_{r}$ and decreasing $T_{w}$. The most deformed molecular rings with the largest $W_{r}$ are almost nonaromatic. The most twisted aromatic and antiaromatic molecular rings sustaining the strongest diatropic and paratropic ring-current strengths, respectively, were also found to be the energetically lowest structures. Our findings do not completely agree with a previous study, ${ }^{1}$ where one concluded that the maximum $\pi$-electronic stability is obtained when maximizing $W_{r}$ for a given $L_{k}$. The energy trends obtained in this work suggest that the energy penalty for increasing $W_{r}$ is larger than the loss of $\pi$-electron energy stabilization favoring strongly twisted molecular structures with small $W_{r}$ values. The present results are supported by an experimental study on a [36] annulene derivative, where the molecular structure with a high twist and a low writhe was found to be the energetically most favorable one. ${ }^{24}$

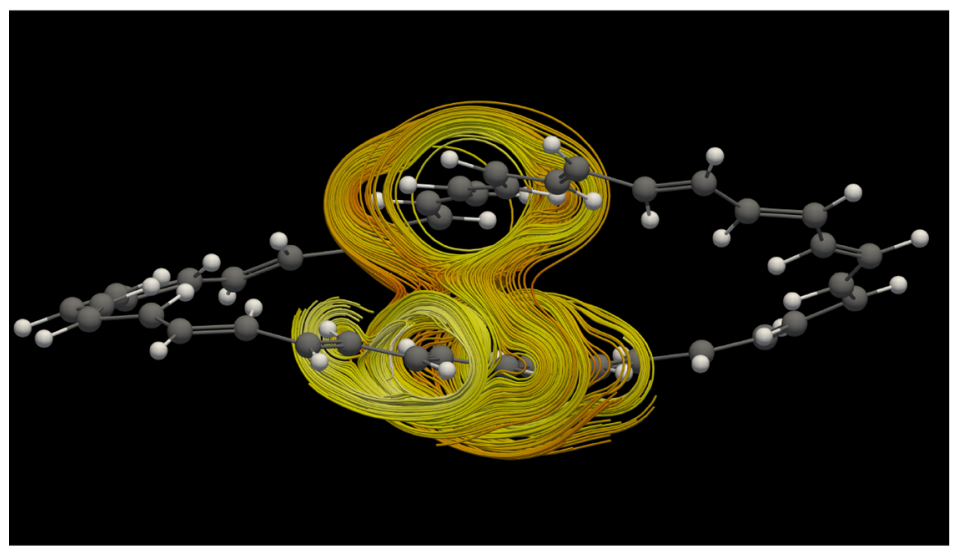

(a)

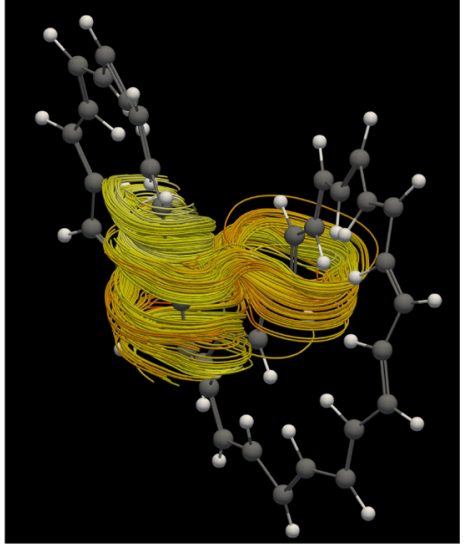

(b)

Figure 6. Current-density vector field illustrated using 3D streamlines for the central region of the doubly charged molecule with $L_{k}=2$ and $T_{w}=$ -0.31 . The top view is shown in (a) and the side view in (b). 


\section{ASSOCIATED CONTENT}

\section{S Supporting Information}

The Supporting Information is available free of charge on the ACS Publications website at DOI: 10.1021/acs.jpclett.8b00440.

Cartesian coordinates, HOMO-LUMO gaps, HOMA indices, current strengths at the B3LYP level, the total energy as a function of the twist number of the investigated molecules, and pictures with line integral convolution representations of the current densities (PDF)

\section{AUTHOR INFORMATION}

\section{Corresponding Authors}

*E-mail: lukas.wirz@helsinki.fi (L.N.W.).

*E-mail: dage.sundholm@helsinki.fi (D.S.).

\section{ORCID $\odot$}

Lukas N. Wirz: 0000-0002-6577-7166

Maria Dimitrova: 0000-0002-0711-3484

Heike Fliegl: 0000-0002-7541-115X

Dage Sundholm: 0000-0002-2367-9277

\section{Notes}

The authors declare no competing financial interest.

\section{ACKNOWLEDGMENTS}

L.N.W. thanks the Alexander von Humboldt Foundation for a Feodor Lynen Research Fellowship. This work has been supported by the Magnus Ehrnrooth Foundation. The Academy of Finland has supported it through Projects 275845 and 297304. H.F. thanks the Norwegian Research Council through the CoE Hylleraas Centre for Quantum Molecular Sciences (Grant No. 262695 and 231571/F20) for support. CSC - IT Center for Science, Finland, the Finnish Grid and Cloud Infrastructure (FGCI), and the Norwegian Supercomputing Program (NOTUR Grant No. NN4654K) are acknowledged for the computational resources.

\section{REFERENCES}

(1) Rappaport, S. M.; Rzepa, H. S. Intrinsically Chiral Aromaticity. Rules Incorporating Linking Number, Twist, and Writhe for HigherTwist Möbius Annulenes. J. Am. Chem. Soc. 2008, 130, 7613-7619.

(2) Schaller, G. R.; Herges, R. Möbius Molecules with Twists and Writhes. Chem. Commun. 2013, 49, 1254-1260.

(3) Klenin, K.; Langowski, J. Computation of Writhe in Modeling of Supercoiled DNA. Biopolymers 2000, 54, 307-317.

(4) Călugăreanu, G. Sur les Classes d'Isotopie des Noeuds Tridimensionnels et Leurs Invariants. Czech. Math. J. 1961, 11, $588-625$.

(5) White, J. H. Self-Linking and Gauss-Integral in Higher Dimensions. Am. J. Math. 1969, 91, 693-728.

(6) Fuller, F. B. The Writhing Number of a Space Curve. Proc. Natl. Acad. Sci. U. S. A. 1971, 68, 815-819.

(7) Heilbronner, E. Hückel Molecular Orbitals of Möbius-Type Conformations of Annulenes. Tetrahedron Lett. 1964, 5, 1923-1928.

(8) Rzepa, H. S. Möbius Aromaticity and Delocalization. Chem. Rev. 2005, 105, 3697-3715.

(9) Herges, R. Topology in Chemistry: Designing Möbius Molecules. Chem. Rev. 2006, 106, 4820-4842.

(10) Hückel, E. Grundzüge der Theorie Ungesättigter und Aromatischer Verbindungen; Verlag Chemie: Berlin, Germany, 1938.

(11) von Eggers Doering, W.; Detert, F. L. Cycloheptatrienylium Oxide. J. Am. Chem. Soc. 1951, 73, 876-877.

(12) Breslow, R. Antiaromaticity. Acc. Chem. Res. 1973, 6, 393-398.
(13) Pople, J. A. Molecular Orbital Theory of Aromatic Ring Currents. Mol. Phys. 1958, 1, 175-180.

(14) McWeeny, R. Ring Currents and Proton Magnetic Resonance in Aromatic Molecules. Mol. Phys. 1958, 1, 311-321.

(15) Pople, J. A.; Untch, K. G. Induced Paramagnetic Ring Currents. J. Am. Chem. Soc. 1966, 88, 4811-4815.

(16) Mallion, R. B. Some Comments on the Use of the "RingCurrent" Concept in Diagnosing and Defining "Aromaticity". Pure Appl. Chem. 1980, 52, 1541-1548.

(17) Wilcox, C. F.; Farley, E. N. Dicyclooctabiphenylene. Synthesis and Properties. J. Am. Chem. Soc. 1984, 106, 7195-7200.

(18) Fowler, P. W.; Rzepa, H. S. Aromaticity Rules for Cycles with Arbitrary Numbers of Half-Twists. Phys. Chem. Chem. Phys. 2006, 8, $1775-1777$.

(19) Castro, C.; Isborn, C. M.; Karney, W. L.; Mauksch, M.; Schleyer, P. v. R. Aromaticity with a Twist: Möbius [4n]Annulenes. Org. Lett. 2002, 4, 3431-3434.

(20) Rzepa, H. S. A. Double-Twist Möbius-Aromatic Conformation of [14]Annulene. Org. Lett. 2005, 7, 4637-4639.

(21) Herges, R. Aromatics with a Twist. Nature 2007, 450, 36-37.

(22) Stępień, M.; Latos-Grażyński, L.; Sprutta, N.; Chwalisz, P.; Szterenberg, L. Expanded Porphyrin with a Split Personality: A Hückel-Möbius Aromaticity Switch. Angew. Chem., Int. Ed. 2007, 46, 7869-7873.

(23) Wannere, C. S.; Rzepa, H. S.; Rinderspacher, B. C.; Paul, A.; Allan, C. S. M.; Schaefer, H. F., III; Schleyer, P. v. R. The Geometry and Electronic Topology of Higher-Order Charged Möbius Annulenes. J. Phys. Chem. A 2009, 113, 11619-11629.

(24) Mohebbi, A.; Mucke, E.-K.; Schaller, G.; Köhler, F.; Sönnichsen, F.; Ernst, L.; Näther, C.; Herges, R. Singly and Doubly Twisted [36]Annulenes: Synthesis and Calculations. Chem. - Eur. J. 2010, 16, $7767-7772$.

(25) McKee, W. C.; Wu, J. I.; Rzepa, H. S.; Schleyer, P. v. R. A Hückel Theory Perspective on Möbius Aromaticity. Org. Lett. 2013, $15,3432-3435$

(26) Vosko, S. H.; Wilk, L.; Nusair, M. Accurate Spin-Dependent Electron Liquid Correlation Energies for Local Spin-Density Calculations - a Critical Analysis. Can. J. Phys. 1980, 58, 1200-1211.

(27) Perdew, J. P. Density-Functional Approximation for the Correlation Energy of the Inhomogeneous Electron Gas. Phys. Rev. B: Condens. Matter Mater. Phys. 1986, 33, 8822-8824.

(28) Becke, A. D. Density-Functional Exchange-Energy Approximation with Correct Asymptotic Behavior. Phys. Rev. A: At., Mol., Opt. Phys. 1988, 38, 3098-3100.

(29) Ahlrichs, R.; Bär, M.; Häser, M.; Horn, H.; Kölmel, C. Electronic Structure Calculations on Workstation Computers: The Program System TURBOMOLE. Chem. Phys. Lett. 1989, 162, 165169. current version: see http://www.turbomole.com.

(30) Furche, F.; Ahlrichs, R.; Hättig, C.; Klopper, W.; Sierka, M.; Weigend, F. Turbomole. WIREs Comput. Mol. Sci. 2014, 4, 91-100.

(31) Schäfer, A.; Horn, H.; Ahlrichs, R. Fully Optimized Contracted Gaussian-Basis Sets for Atoms Li to Kr. J. Chem. Phys. 1992, 97, 25712577.

(32) Treutler, O.; Ahlrichs, R. Efficient Molecular Numerical Integration Schemes. J. Chem. Phys. 1995, 102, 346.

(33) Becke, A. D. A New Mixing of Hartree-Fock and Local DensityFunctional Theories. J. Chem. Phys. 1993, 98, 1372-1377.

(34) Lee, C.; Yang, W.; Parr, R. G. Development of the Colle-Salvetti Correlation-Energy Formula Into a Functional of the Electron Density. Phys. Rev. B: Condens. Matter Mater. Phys. 1988, 37, 785-789.

(35) Häser, M.; Ahlrichs, R.; Baron, H. P.; Weis, P.; Horn, H. Direct Computation of 2nd-order SCF Properties of Large Molecules on Workstation Computers with an Application to Large Carbon Clusters. Theoret. Chim. Acta 1992, 83, 455-470.

(36) Weigend, F.; Ahlrichs, R. Balanced Basis Sets of Split Valence, Triple Zeta Valence and Quadruple Zeta Valence Quality for H to Rn: Design and Assessment of Accuracy. Phys. Chem. Chem. Phys. 2005, 7, 3297-3305. 
(37) Jusélius, J.; Sundholm, D.; Gauss, J. Calculation of Current Densities using Gauge-Including Atomic Orbitals. J. Chem. Phys. 2004, 121, 3952-3963.

(38) Taubert, S.; Sundholm, D.; Jusélius, J. Calculation of SpinCurrent Densities Using Gauge-Including Atomic Orbitals. J. Chem. Phys. 2011, 134, 054123.

(39) Fliegl, H.; Taubert, S.; Lehtonen, O.; Sundholm, D. The Gauge Including Magnetically Induced Current Method. Phys. Chem. Chem. Phys. 2011, 13, 20500-20518.

(40) Sundholm, D.; Fliegl, H.; Berger, R. J. Calculations of Magnetically Induced Current Densities: Theory and Applications. WIREs Comput. Mol. Sci. 2016, 6, 639-678.

(41) Fliegl, H.; Valiev, R. R.; Pichierri, F.; Sundholm, D. Theoretical Studies as a Tool for Understanding the Aromatic Character of Porphyrinoid Compounds. Chem. Modell. 2018, 14, 1-42.

(42) Taubert, S.; Sundholm, D.; Pichierri, F. Magnetically Induced Currents in Bianthraquinodimethane-Stabilized Möbius and Hückel [16]Annulenes. J. Org. Chem. 2009, 74, 6495-6502.

(43) Fliegl, H.; Sundholm, D.; Taubert, S.; Pichierri, F. Aromatic Pathways in Twisted Hexaphyrins. J. Phys. Chem. A 2010, 114, 71537161.

(44) Fliegl, H.; Sundholm, D.; Pichierri, F. Aromatic Pathways in Mono- and Bisphosphorous Singly Möbius Twisted [28] and [30] Hexaphyrins. Phys. Chem. Chem. Phys. 2011, 13, 20659-20665.

(45) Gnuplot. http://www.gnuplot.info/ (2018).

(46) Humphrey, W.; Dalke, A.; Schulten, K. VMD: Visual Molecular Dynamics. J. Mol. Graphics 1996, 14, 33-38.

(47) (a) Ahrens, J.; Geveci, B.; Law, C. Paraview: An End-User Tool for Large Data Visualization, Visualization Handbook; Elsevier, 2005.

(b) Paraview. http://www.paraview.org (2018).

(48) GIMP: GNU Image Manipulation Program. http://www.gimp. org (2017).

(49) Cabral, B.; Leedom, L. C. Imaging Vector Fields Using Line Integral Convolution. Proceedings of the 20th Annual Conference on Computer Graphics and Interactive Techniques, New York, 1993; pp 263-270.

(50) Becke, A. D. Density-Functional Thermochemistry. III. The Role of Exact Exchange. J. Chem. Phys. 1993, 98, 5648-5652.

(51) Valiev, R. R.; Fliegl, H.; Sundholm, D. Optical and Magnetic Properties of Antiaromatic Porphyrinoids. Phys. Chem. Chem. Phys. 2017, 19, 25979-25988.

(52) Valiev, R. R.; Fliegl, H.; Sundholm, D. Closed-Shell Paramagnetic Porphyrinoids. Chem. Commun. 2017, 53, 9866-9869.

(53) Kumar, C.; Fliegl, H.; Sundholm, D. The Relation Between Ring Currents and Hydrogenation Enthalpies for Assessing the Degree of Aromaticity. J. Phys. Chem. A 2017, 121, 7282-7289.

(54) Fliegl, H.; Sundholm, D.; Taubert, S.; Jusélius, J.; Klopper, W. Magnetically Induced Current Densities in Aromatic, Antiaromatic, Homoaromatic, and Nonaromatic Hydrocarbons. J. Phys. Chem. A 2009, 113, 8668-8676.

(55) Krygowski, T. M.; Cyrañski, M. K.; Czarnocki, Z.; Häfelinger, G.; Katritzky, A. R. Aromaticity: A Theoretical Concept of Immense Practical Importance. Tetrahedron 2000, 56, 1783-1796.

(56) Julg, A.; François, P. Recherches sur la Géométrie de Quelques Hydrocarbures Non-Alternants: Son Influence sur les Énergies de Transition, une Nouvelle Définition de l'Aromaticité. Theoret. Chim. Acta 1967, 8, 249-259. 